ARTICLE

DOI: $10.1038 / \mathrm{s} 41467-018-05034-6$

\title{
Ultrathin and multicolour optical cavities with embedded metasurfaces
}

Amr M. Shaltout ${ }^{1,2}$, Jongbum Kim (1D ${ }^{1,3}$, Alexandra Boltasseva1, Vladimir M. Shalaev ${ }^{1}$ \& Alexander V. Kildishev (D) ${ }^{1}$

Over the past years, photonic metasurfaces have demonstrated their remarkable and diverse capabilities in advanced control over light propagation. Here, we demonstrate that these artificial films of deeply subwavelength thickness also offer new unparalleled capabilities in decreasing the overall dimensions of integrated optical systems. We propose an original approach of embedding a metasurface inside an optical cavity-one of the most fundamental optical elements-to drastically scale-down its thickness. By modifying the Fabry-Pérot interferometric principle, this methodology is shown to reduce the metasurface-based nanocavity thickness below the conventional $\lambda /(2 n)$ minimum. In addition, the nanocavities with embedded metasurfaces can support independently tunable resonances at multiple bands. As a proof-of-concept, using nanostructured metasurfaces within 100-nm nanocavities, we experimentally demonstrate high spatial resolution colour filtering and spectral imaging. The proposed approach can be extrapolated to compact integrated optical systems on-a-chip such as VCSEL's, high-resolution spatial light modulators, imaging spectroscopy systems, and bio-sensors.

\footnotetext{
${ }^{1}$ School of Electrical \& Computer Engineering and Birck Nanotechnology Center, Purdue University, West Lafayette, Indiana 47907, USA. ${ }^{2}$ Geballe Laboratory for Advanced Materials, Stanford University, Stanford, CA 94305, USA. ${ }^{3}$ Institute for Research in Electronics and Applied Physics, University of Maryland, College Park, Maryland 20742, USA. These authors contributed equally: Amr M. Shaltout, Jongbum Kim. Correspondence and requests for materials should be addressed to A.V.K. (email: kildishev@purdue.edu)
} 
$\mathrm{P}=$ hotonic metasurfaces ${ }^{1-6}$ have enabled a new paradigm of controlling light by passing it through planar arrays of nanostructured antennas to induce a swift change in phase and/or polarization. Consequently, photons propagating through metasurfaces could be 'processed' to undergo a change to their momentum, angular momentum, and/or spin state. This has led to a relaxation of Snell's $l_{a w}^{2}$, a pivotal relation in optics, and has enabled an entirely new family of flat optical elements. These elements can provide diverse functionalities for a plethora of applications including light bending devices ${ }^{2,7}$, flat lenses ${ }^{8-11}$, holograms ${ }^{12-17}$, wave-plates ${ }^{18-22}$, as well as devices with chiral $^{23-28}$ and bianisotropic ${ }^{29,30}$ optical responses. A typical metasurface generates its functional output in the far field, which requires a separation distance between the metasurface and the location of the detected output. Therefore, even though a given metasurface could be ultrathin, an integrated optical system that requires cascading of the metasurface with another optical component may not fully utilize its capability to reduce the overall dimensions of the system.

Optical cavities are the major components of lasers and other numerous interferometric optical devices, and minimizing the cavity dimensions below the diffraction limit is the key for many applications, including realization of nano-lasers ${ }^{31-33}$, and the enhancement of spontaneous emission rate due to the Purcell effect $^{34}$. This enhancement, which is useful for single-photon sources $^{35,36}$ and low threshold lasing ${ }^{37-39}$ and is inversely proportional to the volume of the cavity, has motivated nanophotonics researchers in quest of solutions minimizing the cavity dimensions ${ }^{40-44}$.

Here we show that embedding a metasurface inside a most fundamental optical element-an optical cavity-can substantially reduce the cavity thickness (up to two times). Such significant reduction in the transverse dimension is achieved without changing the optical mode for the sake of cascading and integration with other optical devices. In the following sections, we present a metasurface-based Fabry-Pérot nanocavity arranged of two parallel reflective metal layers and a metasurface sandwiched between them. First, we theoretically study the impact of the metasurface on the resonant condition of nanocavity based upon the interferometric principle. Then, we discuss the structure fabrication and the experimental results, which show that a 100$\mathrm{nm}$ nanocavity supports resonances within the wavelength band from 500 to $800 \mathrm{~nm}$ and demonstrate successful application of the fabricated nanocavity samples as on-a-chip ultrathin colour filters. In addition to reducing the cavity thickness, the metasurface can also add another degree of freedom to control the cavity resonance. With the introduction of the metasurface, the cavity resonance can be tuned through adjusting the parameters of the metasurface-not only by adjusting the cavity thickness. This is especially useful for building multiple cavities of the same thickness on a planar chip resonating at different wavelengths. These additional degrees of freedom also allow us to obtain dualband resonances or even produce coloured images by using a single planar nanocavity of the same thickness by simply choosing the appropriate design for the internal metasurface.

\section{Results}

Theoretical formulation. The resonant condition of a conventional Fabry-Pérot cavity made of two reflecting mirrors occurs when the round-trip optical phase inside the cavity is an integer multiple of $2 \pi$. This imposes a limitation on the cavity thickness to accumulate the required phase. Our approach to overcome this limitation is to place a metasurface inside the two mirrors which induces a strong phase shift and compensates for the reduced accumulated phase in the rest of the cavity.
Figure 1(a) is a schematic of the nanocavity composed of two silver layers as reflecting surfaces, a silver-based metasurface separated from the bottom Ag mirror by an alumina spacer layer, and a polymer which fills the rest of the cavity. Figure $1(b, c)$ demonstrate the cross section of a Fabry-Pérot cavity without and with the embedded metasurfaces, respectively. At resonance, the round-trip phase shift inside the cavity satisfies the following formulas in case of utilizing a perfect mirror:

$$
\begin{aligned}
& \frac{4 \pi n L}{\lambda}=2 \pi m, \text { without metasurface, } \\
& \frac{4 \pi n L}{\lambda}+\varphi_{\mathrm{ms}}=2 \pi m, \text { with metasurface }
\end{aligned}
$$

where $L$ is the thickness of the cavity, $n$ is the refractive index of the filling material, $\varphi_{\mathrm{ms}}$ is the phase shift induced by the metasurface, and $m=1,2, \ldots$. The conventional formula implies that for the metasurface-free cavity, a minimum cavity thickness $L_{\text {min }}$ is on the order of $\lambda /(2 n)$ when $m=1$. In the case of an embedded metasurface, the induced phase $\varphi_{\mathrm{ms}}$ reduces the required phase $4 \pi L / \lambda$ for the rest of the cavity, and hence enables $L_{\text {min }}$ to go below $\lambda /(2 n)$. For practical purposes of operating with a very thin silver layer, a model, which includes an extra phase term induced by the Ag mirror, is more accurate ${ }^{45}$

$$
\begin{aligned}
& \frac{4 \pi n L}{\lambda}+\varphi_{\mathrm{Ag}, 1}+\varphi_{\mathrm{Ag}, 2}=2 \pi m, \text { without metasurface } \\
& \frac{4 \pi n L}{\lambda}+\varphi_{\mathrm{Ag}, 1}+\varphi_{\mathrm{ms}}=2 \pi m, \text { with metasurface }
\end{aligned}
$$

where $\varphi_{\mathrm{Ag}, 1}$ and $\varphi_{\mathrm{Ag}, 2}$ are the extra phase shifts induced by the top and bottom silver mirrors, respectively, for $m=1,2, \ldots$. In case of having the metasurface embedded with the bottom mirror, the term $\varphi_{\mathrm{Ag}, 2}$ should be replaced by $\varphi_{\mathrm{ms}}$. In the case of $15-\mathrm{nm}$ silver Ag mirror, studies have shown ${ }^{45}$ that phase shift induced by silver mirrors would make the minimum cavity thickness $L_{\min }$ on the order of $0.35 \lambda / n$.

The underlying physical mechanism that induces the strong phase shift by the metasurface is the excitation of gap-plasmon resonators ${ }^{46-48}$ and slow plasmonic modes that propagate inside

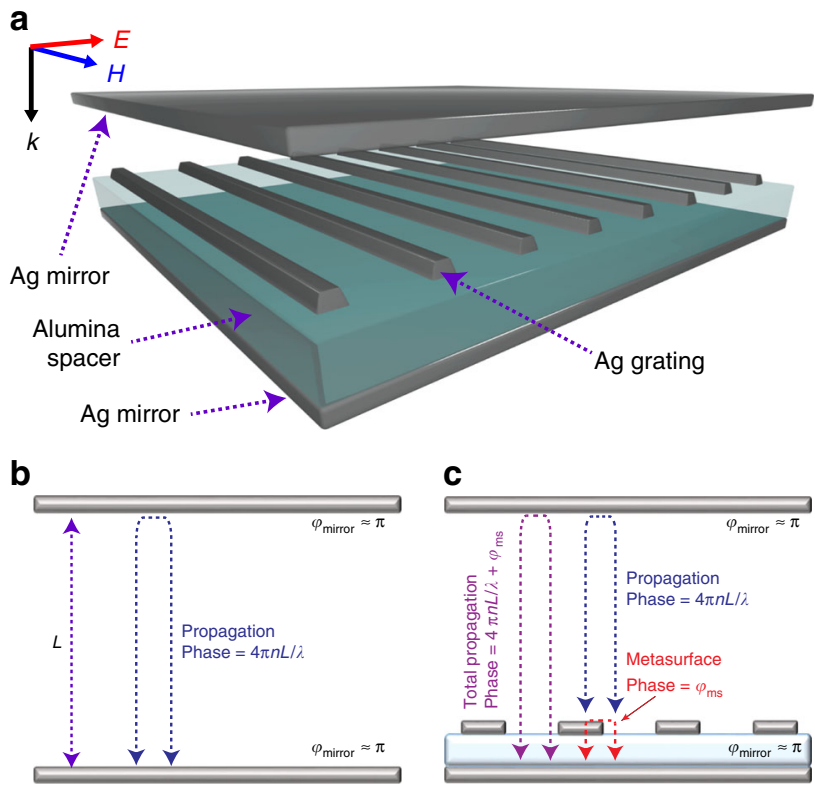

Fig. 1 Design and concept of metasurface-based nanocavity. a Schematic of the metasurface-based nanocavity. b, c Comparison in phase and resonance conditions between: $\mathbf{b}$ conventional Fabry-Pérot resonator that utilizes two parallel mirrors and $\mathbf{c}$ resonator with a reflecting metasurface placed in between the two mirrors 
a

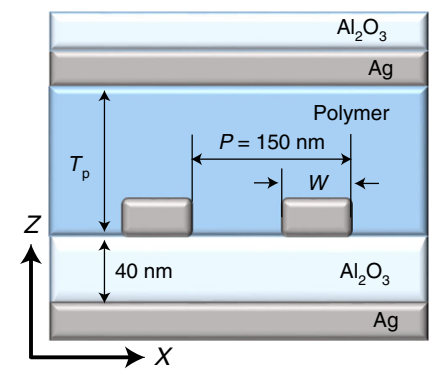

b
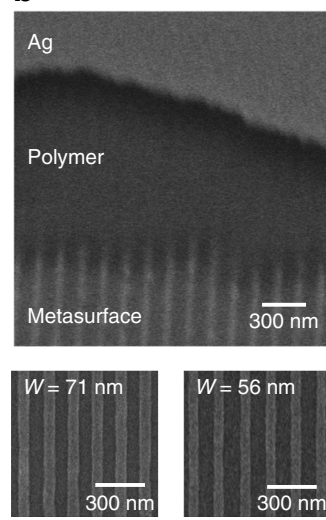

c

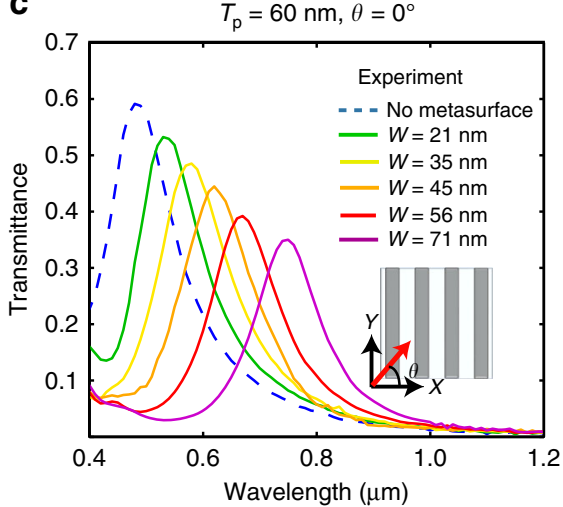

e

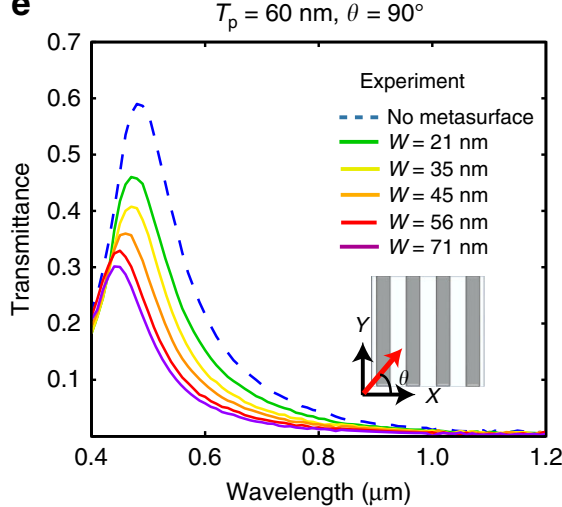

d

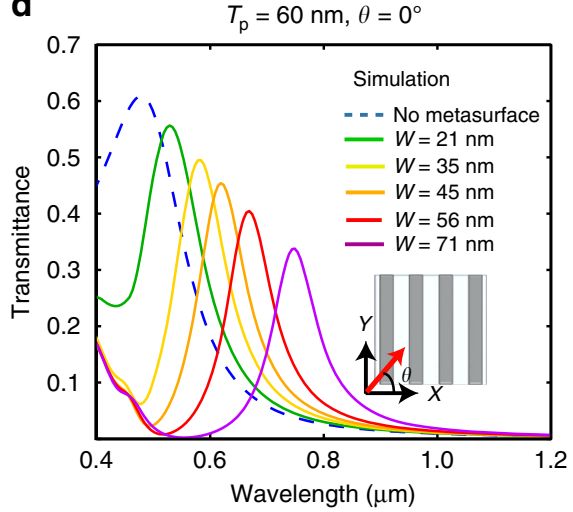

f

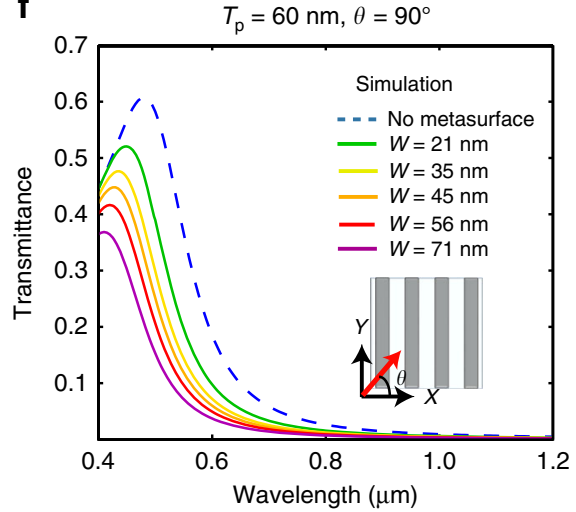

Fig. 2 Spectral transmission of Ag based nanocavities with and without the metasurface. a Cross-sectional schematic of the metasurface-based nanocavity.

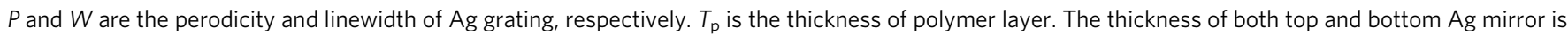
$15 \mathrm{~nm}$ and the thickness of grating is $22 \mathrm{~nm}$. b $30^{\circ}$ tilted view and Top view of FE SEM (Field Emission Scanning Electron Microscopy) image of fabricated nanocavity. Experiment (c, e) and simulation $(\mathbf{d}, \mathbf{f})$ of transmission spectra of nanocavity with $60 \mathrm{~nm}$ thick polymer spacer (inset in $\mathbf{c}$ : the schematic of polarization direction, $\theta$ is the angle of polarization of incident wave): $\mathbf{c}, \mathbf{d} \theta=0^{\circ}$ and $\mathbf{e}, \mathbf{f} \theta=90^{\circ}$

the alumina spacer layer between the bottom silver layer and the metasurface structure. These gap-plasmon modes can create large phase shifts for the wavefronts propagating inside the cavity. The phase shifts induced by the gap-plasmons can be controlled by adjusting the lateral dimensions of the metasurface Ag nano-strip layer. For example, increasing the nano-strip width makes the gap-plasmons propagate over a greater lateral distance and acquire additional phase, thereby decreasing the minimum cavity phase shift required for the Fabry-Pérot resonance ${ }^{48}$.

Implementation and experimental results. Figure 2(a) shows a cross-sectional schematic of the nanocavity. It is implemented with 15-nm-thick Ag mirrors, a 40-nm-thick Alumina spacer, 22$\mathrm{nm}$-thick Ag metasurface gratings, and a polymer thickness in the rest of the cavity of $60 \mathrm{~nm}$, which makes the whole cavity thickness between the two mirrors to be around $100 \mathrm{~nm}$. The structure has a lateral period of $150 \mathrm{~nm}$. Figure 2(b) shows a $30^{\circ}$ tilted view of the nanocavity where a cross-sectional shear is introduced through the upper Ag layer and the polymer layer till the metasurface layer. Five different samples are fabricated with different metasurface widths of $W=21,35,45,56$, and $71 \mathrm{~nm}$. All the samples are fabricated on the same chip, and hence, they hold the same cavity thickness and any difference they exhibit in their optical performance is attributed to the different width $(W)$ in their metasurface structure. (See Methods section for the detailed fabrication and measurement procedure).

To demonstrate the impact of the metasurface on the nanocavity, spectral transmission measurements are conducted on the nanocavities with and without the metasurface. Figure 2(c, d) demonstrate spectral measurements and the transmission data respectively, obtained from finite-element simulation with the incident polarization being perpendicular to the Ag metasurface gratings, since this polarization enables gap-plasmon excitation across the width of the meta-gratings ${ }^{48}$. Comparing the spectral data without a metasurface (dashed line) to those of metasurfacebased nanocavities, we conclude that the metasurface makes the resonant wavelength longer across the visible and NIR spectra. A numerical study of the phase shift induced by the metasurface is included in Supplementary Fig. 1. The study provides that increasing the metasurface $\mathrm{Ag}$ grating dimensions increase the induced phase shift $\left(\varphi_{\mathrm{ms}}\right)$ inside the metasurface. It is demonstrated in Supplementary Fig. 1 that $\varphi_{\mathrm{ms}}$ has a broadband response. This is owing to the fact that the retardation phase in the gap-plasmon structure is not a resonant effect. The relatively large $40-\mathrm{nm}$ thickness of the spacer is responsible for coupling most of the optical power to the gap-plasmon mode. If the spacer thickness were too narrow, the light would only couple to the gap-plasmon mode when Fabry-Pérot resonance overlapped with gap-plasmon resonance creating a hybrid mode $\mathrm{e}^{49}$, which would be different from the effect required in our case, moreover it would demand very careful adjustment of the structure to match the Fabry-Pérot and plasmonic resonances, otherwise, it would not operate.

According to Equation (2), increasing $\varphi_{\mathrm{ms}}$ causes an increase in wavelength $\lambda$ for the equation to be satisfied. Therefore, resonant wavelength increases as the Ag gratings width increases across the values $W=21,35,45,56$, and $71 \mathrm{~nm}$. All these samples resonating at different wavelengths are having the same cavity thickness. The structured metasurface adds a new degree of freedom of controlling resonant wavelength other than the cavity 


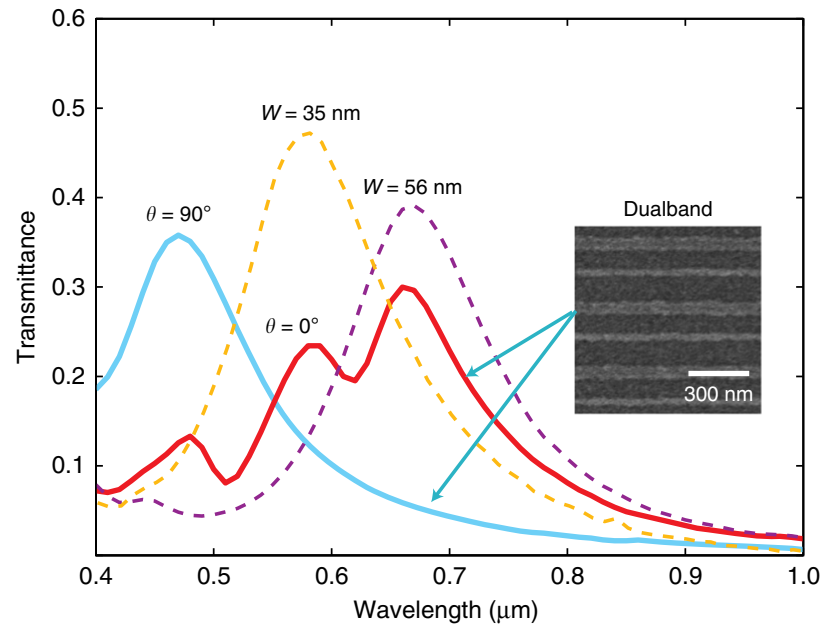

Fig. 3 Spectra transmission of dualband nanocavity. Experimentally obtained transmission spectra of dualband nanocavity with 60-nm-thick polymer spacer: the dualband metasurface consists of two different $\mathrm{Ag}$ gratings with $35 \mathrm{~nm}$ and $56 \mathrm{~nm}$ linewidth

thickness. On the other hand, the impact of the metasurface on the resonant wavelength when light polarization is parallel to the gratings is not significant since this polarization is poorly coupled to the gap-plasmonic mode. This reflects in the spectral measurement and simulation data shown in Fig. 2(e, f). The simulation data is well-matched with the experimental data; however, the simulated transmission data within the wavelength range from 400 to $500 \mathrm{~nm}$ are higher, since the utrathin Ge adhesion layer is excluded from the simulation domain ${ }^{50}$. Supplementary Fig. 2 demonstrates the simulation of the reflection and the absorption spectra to complement the transmission study in Fig. 2(d). The dominant loss mechanism is attributed to the absorption in the metal mirror that can be shown by comparing the absorption of devices without and with the metasurface. The increase of absorption due to the insertion of the metasurface is insignificant (below 10\%). Despite the relatively low additional loss due to the metasurface, it has a wideband effect due to the strong coupling efficiency in and out of the gap-plasmon mode. Another experimental study on a different metasurface-based nanocavity of polymer thickness 240 $\mathrm{nm}$ is demonstrated in Supplementary Fig. 3. It shows that the impact of the metasurface in increasing the resonant wavelength is effective in fundamental resonant mode as well as higher order modes.

Dualband resonance. The formula of Equation (2) as well as the measurements in Fig. 2(c) indicates that metasurface-based nanocavities are dependent on both the cavity thickness $L$ and the metasurface induce phase $\varphi_{\mathrm{ms}}$, making the resonance controllable via two independent degrees of freedom. If the metasurface structure is designed such that it can induce a strong phase shift $\varphi_{\mathrm{ms}}$ at more than one wavelength, then multiple resonances can occur at different wavelengths that can be independently controlled through careful design of the metasurface. This enables a new technique of obtaining multi-band resonance other than having different higher modes standing waves across the cavity thickness (modes with $m>1$ ).

To obtain a dualband resonance, we implement a nanocavity where the metasurface is composed of alternating grating widths of 35 and $56 \mathrm{~nm}$ as shown in Fig. (3). We have had two samples, each of them has its grating width designed with only one of these two values. They were part of the samples whose results are shown in Fig. 2. We demonstrate the individual response of each of these two samples in Fig. 3 along with the sample implemented with the alternating grating. The corresponding simulation results are presented in Supplementary Fig. 4. The sample with the hybrid Ag grating dimensions have demonstrated dualband resonances, which coincide with the two individual resonances obtained by the other two samples. This can be attributed to the metasurface's hybrid dimensions which will support gapplasmonic modes at both wavelengths supported by grating widths of 35 and $56 \mathrm{~nm}$. The small peak at a wavelength similar to the resonance of the cross-polarized light is attributed to the strong absorption of Ge layer within the wavelength from $400 \mathrm{~nm}$ to $500 \mathrm{~nm}$ (See Supplementary Fig. 4). The different resonant wavelengths obtained through this technique can be independently tuned by a separate adjustment of different grating widths.

Ultrathin colour filtering and imaging. One of the straightforward and promising applications of optical cavities is spectral filtering. The polarization sensitive colour filtering can be obtained from the metasurface embedded nanocavity due to its narrow linewidth and broad tunability of resonance in the visible range. In addition, from white light through corresponding shaping of nanocavities, colour images can be generated in planar surface which make this device easy to be integrated with other optical components. With the same nanocavity thickness of 100 $\mathrm{nm}$ as in previously shown results, we implement six different nanocavities, each of them is shaped to one of the letters of the word PURDUE, with each letter having a dimension of $500 \mu \mathrm{m} \times$ $420 \mu \mathrm{m}$. The letters $\mathrm{P}, \mathrm{U}, \mathrm{R}, \mathrm{D}, \mathrm{U}$ are shaped with Ag metasurfaces having grating widths of $W=21,35,45,56$, and $71 \mathrm{~nm}$, respectively, which are the same dimensions of metasurfaces as demonstrated in Fig. 2. Letter E is implemented using a metasurface with alternating gratings of 35 and $56 \mathrm{~nm}$ similar to the one in Fig. 3. Broadband light from a white lamp is filtered through a polarizer, and applied to the sample, and multicoloured images are captured from the other side of the sample. Figure 4(a) demonstrates the colour images obtained for different polarization directions of incident light with $0^{\circ}$ corresponding to perpendicular polarization, and $90^{\circ}$ to parallel polarization with respect to Ag gratings. The coloured image is in good agreement with the spectral data in Figs 2-3. First, the part of the cavity outside the metasurface filters a sky-blue colour that corresponds to its spectral data. The images of samples $1,2,3$, and 4 for $0^{\circ}$ polarization have their resonant wavelengths pushed to the spectra of green, yellow, orange, and red, respectively. For sample 5 , the spectral data is in the NIR outside the visible spectrum explaining the dark appearance of the letter E. For sample 6, there is a dualband (such as the one in Fig. 3) which is a hybridization of two colours leading to the generation of the brown colour for letter F. For all the six samples, the $90^{\circ}$-polarized light generates a blue colour similar to the one in the metasurface-free background with a smaller intensity. This is also in agreement with the experimental data shown in Fig. 2. For other polarizations between $0^{\circ}$ and $90^{\circ}$, the obtained spectrum is a linear combination of the horizontally and vertically polarized spectra. Figure 4 (c, d) present the measured spectra for these polarizations for sample 4 and 6 . In order to understand what colour will be the result of this linear superposition, we need to use the standard 1913 CIE (Commission internationale de l'éclairage) colour map that maps the spectral data onto a corresponding colour. This map exhibits the property that a linear superposition of two spectra will correspond to the linear superposition of their corresponding locations on the map (i.e., along the line connecting the two locations). The map location of the six samples for horizontal and vertical polarizations were obtained (The methods section explains the tools used to obtain them). The line 


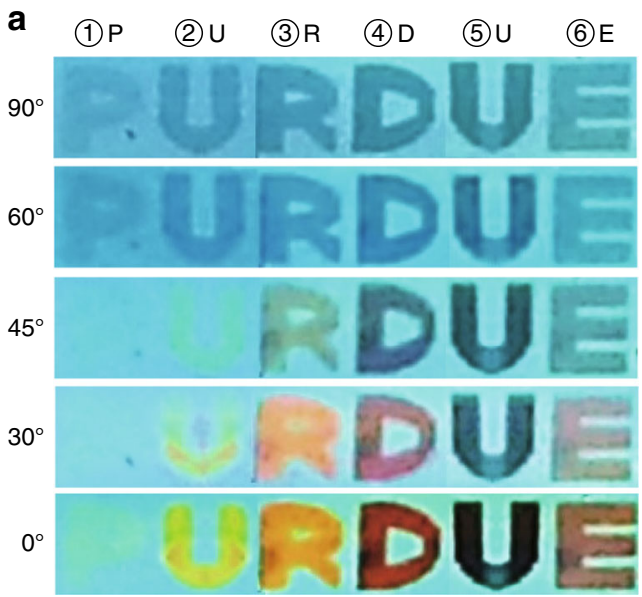

b
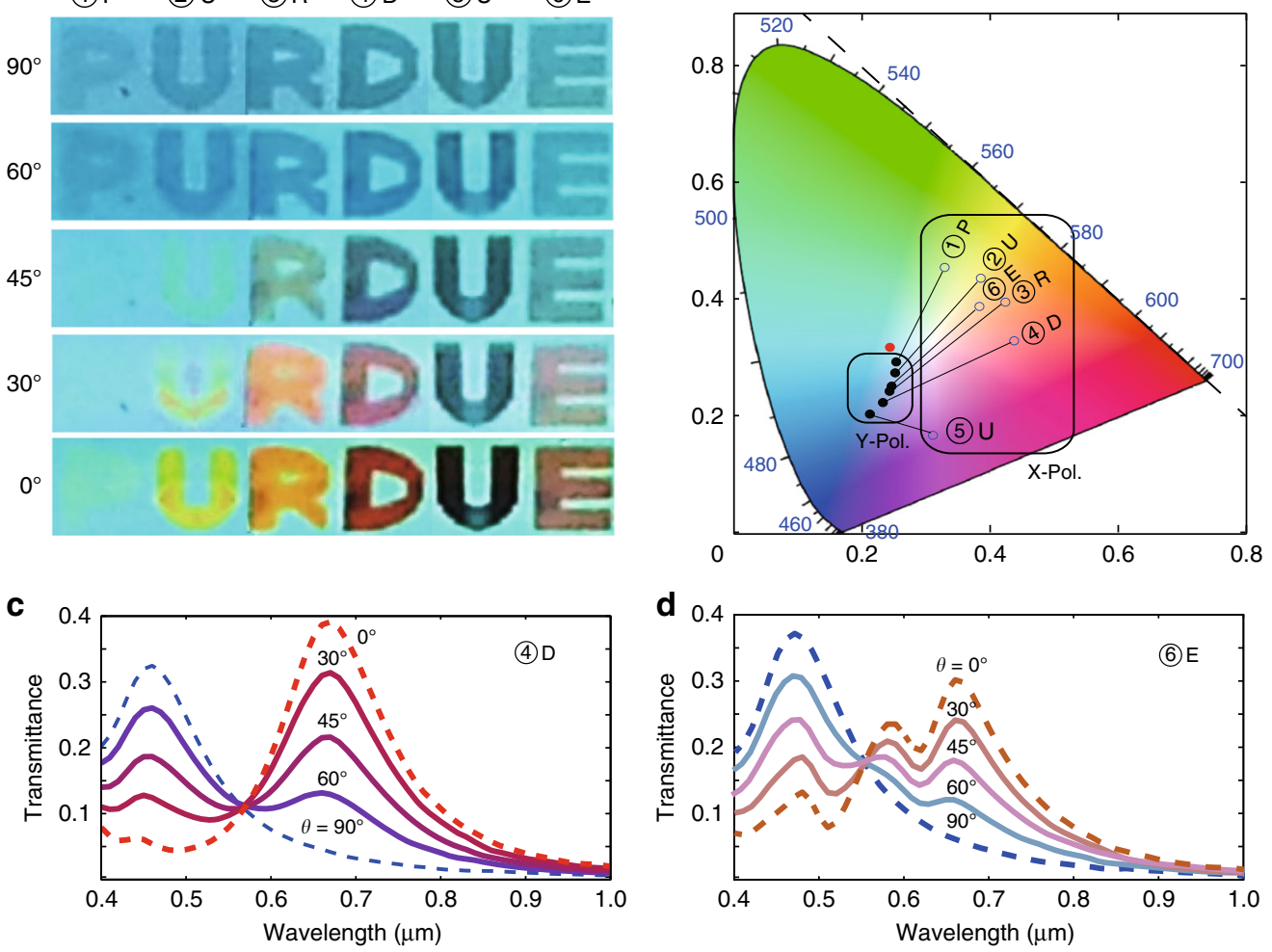

Fig. 4 Bandpass colour filtering with the metasurface-based nanocavities in the visible range. a Colour image of the nanocavity with different linewidth. White light illuminates the sample via a linear polarizer. The angle of polarization is varied from $0^{\circ}$ to $90^{\circ}$. $\mathbf{b}$ The variation of colour with angle of polarization is analyzed with the CIE 1931 colour mapping. The red dot represents the background colour. c, $\mathbf{d}$ The transmission spectra of nanocavity at different angle of polarization: $\mathbf{c}$ for sample 4 (letter 'D') and $\mathbf{d}$ for sample 6 (letter ' $E$ ')

connecting the two locations in the map should reflect the colours generated when the polarization is rotated between $0^{\circ}$ and $90^{\circ}$. Figure $4(\mathrm{a}, \mathrm{b})$ indicate a good agreement between the colour images and the corresponding position on the 1931 CIE colour map. Previous implementations of colour filtering using metasurfaces $^{51-53}$ depend mainly on plasmonic spectral absorption providing a band-stop filtering operation. This can be useful with colour displays working with C-M-Y-K basis, while our metasurface-based Fabry-Pérot structure provides bandpass colour filtering, which is more compatible with the most common RG-B colour displays.

In addition to compactness, metasurface-based nanocavities offer more degrees of freedom to the design of colour filters and displays with respect to conventional metal-insulator-metal (MIM) cavities. For example, they can provide dualband operation which is beneficial for mixing up colours. Another key advantage is the possibility of building different cavities with different resonant wavelengths/colours within the same planar structure in one fabrication run. To demonstrate this capability, we realize the word 'PURDUE' with letters in colour on a single substrate by using a single EBL step allowing to build all letters simultaneously by varying the lateral dimensions of the metasurfaces. If similar cavities were to be realized using separate MIM cavities it would require six different cavity thicknesses or different filling materials, entailing a fabrication process, which would be substantially more challenging and less robust. In addition, the proposed design can be easily integrated with modern highly efficient broadband light emitters, providing a straightforward approach to building a multicolour display pixel on a single emitter.

\section{Discussion}

A unique methodology of utilizing metasurfaces to achieve miniaturized nanophotonic devices is introduced. Metasurfacebased nanocavities are proposed with dimensions that can go below the conventional diffraction limit of $\lambda /(2 n)$ in the material between the cavity mirrors. Embedding metasurfaces inside cavities provides other degrees of freedom for controlling resonant wavelengths in addition to adjusting the cavity thickness or dielectric material index. Several cavities are implemented on the same planar chip with the same thickness and we are able to obtain different resonant wavelengths through controlling the width of nanostructured metasurface elements. Multi-band filtering, colouration, and imaging is also made possible inside the same cavity by controlling the degrees of freedom provided by the metasurface. The proposed approach could have key impact for many optical applications including, but not limited to, nanolasers and ultra-compact filters, as well as other interesting physical applications related to spontaneous emission enhancement based on the Purcell effect, cavity quantum electrodynamics, structural colouration, as well as plasmonics enhanced optoelectronic devices, including spatial light modulators with subwavelength resolution, spectroscopic imaging, and bio-sensing systems. Nano-lasers can be built by using a gain medium in the spacer and/or polymer layer. In addition, this work has the potential in future designs of dynamically controlled nanocavities utilizing tunable spacer layer materials, such as transparent conducting oxides, in which the free carrier concentration can be electrically or optically modulated. On the other hand, using a highly nonlinear spacer could be beneficial for optical limiting devices and saturable absorbers ${ }^{54}$. 


\section{Methods}

Sample preparation. The designed structure is compatible with nanofabrication techniques. 15 -nm-thick $\mathrm{Ag}$ and 40 -nm-thick $\mathrm{Al}_{2} \mathrm{O}_{3}$ films were subsequently deposited on the glass substrate by electron beam evaporator. To fabricate $\mathrm{Ag}$ metasurface (grating structure), positive electron beam resist (ZEP $520 \mathrm{~A}$ ) was spin-coated and grating arrays were defined by electron beam lithography (Vistec VB6) followed by lift-off process. On top of the patterned resist, 22-nm Ag film was deposited with a 3-nm Ge layer used to improve adhesion, and the sample was dipped in ZDMAC (dimethylacetamide) for $20 \mathrm{~min}$. For planar surface of spacer layer on top of metasurface, we spin-coated $800 \mathrm{~nm}$ PMMA on top of metasurface and etched it down to desired thickness $(60 \mathrm{~nm}$ or $240 \mathrm{~nm}$ ) by reactive ion etching process (Panasonic E620 etcher). The roughness of etched PMMA spacer on top of metasurface is around $3 \mathrm{~nm}$. Finally, 3-nm Ge film and 15- $\mathrm{nm} \mathrm{Ag} \mathrm{film} \mathrm{for} \mathrm{top}$ mirror layer and 40-nm alumina layer for protection of $\mathrm{Ag}$ were deposited on top of PMMA spacer.

Sample characterization. The transmission spectra of fabricated nanocavities were measured using the spectroscopic ellipsometer (J. A. Woollam Co., V-VASE). The light source is a xenon lamp with a broadband Visible to NIR spectrum. The diameter of the incident beam was set to be $400 \mu \mathrm{m}$. The beam sequentially passed through a monochromator, a polarizer, and it is used to expose the sample, and then it's collected by a detector. The colour image was captured with simple optical set-up. The white lamp was used as light source and linear polarizer was placed between lamp and sample. The image was captured with commercial camera in mobile phone (Samsung Galaxy S3) with $\times 4$ magnification. ISO was set to 100 , auto-contrast was off, and the micro lens was attached to camera.

Simulation. We validated the experimental spectra with numerical simulations using a commercially available software (COMSOL Multiphysics, Wave Optics Module) based on the Finite Element Method. 1913 CIE colour map was calculated using Matlab with an open-access code from the Matlab website, https://www. mathworks.com/matlabcentral/fileexchange/29620-cie-coordinate-calculator.

Data availability. The authors declare that the data supporting the findings of this study are available within the paper and its supplementary information file.

Received: 8 May 2017 Accepted: 6 June 2018

Published online: 10 July 2018

\section{References}

1. Bomzon, Z., Biener, G., Kleiner, V. \& Hasman, E. Space-variant Pancharatnam-Berry phase optical elements with computer-generated subwavelength gratings. Opt. Lett. 27, 1141-1143 (2002).

2. $\mathrm{Yu}, \mathrm{N}$. et al. Light propagation with phase discontinuities reflection and refraction. Science 334, 333-337 (2011).

3. Yu, N. \& Capasso, F. Flat optics with designer metasurfaces. Nat. Mater. 13, 139-150 (2014).

4. Kildishev, A. V., Boltasseva, A. \& Shalaev, V. M. Planar photonics with metasurfaces. Science 339, 12320091-12320096 (2013).

5. Genevet, P., Capasso, F., Aieta, F., Khorasaninejad, M. \& Devlin, R. Recent advances in planar optics: from plasmonic to dielectric metasurfaces. Optica $\mathbf{4}$, 139-152 (2017).

6. Mueller, J. P. B., Rubin, N. A., Devlin, R. C., Groever, B. \& Capasso, F. Metasurface polarization optics: independent phase control of arbitrary orthogonal states of polarization. Phys. Rev. Lett. 118, 113901 (2017).

7. Ni, X., Emani, N. K., Kildishev, A. V., Boltasseva, A. \& Shalaev, V. M. Broadband light bending with plasmonic nanoantennas. Science 335, 427 (2012).

8. Aieta, F. et al. Aberration-free ultrathin flat lenses and axicons at telecom wavelengths based on plasmonic metasurfaces. Nano. Lett. 12, 4932-4936 (2012).

9. Chen, X. et al. Dual-polarity plasmonic metalens for visible light. Nat. Commun. 3, 1198 (2012).

10. Hasman, E., Kleiner, V., Biener, G. \& Niv, A. Polarization dependent focusing lens by use of quantized Pancharatnam-Berry phase diffractive optics. Appl. Phys. Lett. 82, 328-330 (2003).

11. Ni, X., Ishii, S., Kildishev, A. V., \& Shalaev, V. M. Ultra-thin, planar, Babinetinverted plasmonic metalenses. Light Sci. Appl. 2, e72 (2013).

12. Chen, W. T. et al. High-efficiency broadband meta-hologram with polarization-controlled dual images. Nano. Lett. 14, 225-230 (2014).

13. Larouche, S., Tsai, Y. J., Tyler, T., Jokerst, N. M. \& Smith, D. R. Infrared metamaterial phase holograms. Nat. Mater. 11, 450-454 (2012).
14. Lin, J., Genevet, P., Kats, M. A., Antoniou, N. \& Capasso, F. Nanostructured holograms for broadband manipulation of vector beams. Nano. Lett. 13, 4269-4274 (2013)

15. Montelongo, Y. et al. Plasmonic nanoparticle scattering for colour holograms. Proc. Natl Acad. Sci. USA 111, 12679-12683 (2014).

16. Ni, X., Kildishev, A. V. \& Shalaev, V. M. Metasurface holograms for visible light. Nat. Commun. 4, 2807 (2013).

17. Zheng, G. et al. Metasurface holograms reaching $80 \%$ efficiency. Nat. Nanotechnol. 10, 308-312 (2015).

18. Ding, F., Wang, Z., He, S., Shalaev, V. M. \& Kildishev, A. V. Broadband highefficiency half-wave plate: a supercell-based plasmonic metasurface approach. ACS Nano 9, 4111-4119 (2015).

19. Pors, A. \& Bozhevolnyi, S. I. Efficient and broadband quarter-wave plates by gap-plasmon resonators. Opt. Express 21, 2942-2952 (2013).

20. Yu, N. et al. A broadband, background-free quarter-wave plate based on plasmonic metasurfaces. Nano. Lett. 12, 6328-6333 (2012).

21. Zhu, Z. H. et al. Metallic nanofilm half-wave plate based on magnetic plasmon resonance. Opt. Lett. 37, 698-700 (2012).

22. Kim, J. et al. Controlling the polarization state of light with plasmonic metal oxide metasurface. ACS Nano 10, 9326-9333 (2016).

23. Papakostas, A. et al. Optical manifestations of planar chirality. Phys. Rev. Lett. 90, 107404 (2003)

24. Plum, E. et al. Metamaterials: optical activity without chirality. Phys. Rev. Lett. 102, 113902 (2009).

25. Plum, E. et al. Metamaterial with negative index due to chirality. Phys. Rev. $B$ Condens. Matter Mater. Phys. 79, 035407 (2009).

26. Prosvirnin, S. L. \& Zheludev, N. I. Polarization effects in the diffraction of light by a planar chiral structure. Phys. Rev. E Stat. Nonlinear, Soft Matter Phys. 71, 037603 (2005)

27. Shaltout, A., Liu, J., Shalaev, V. M. \& Kildishev, A. V. Optically active metasurface with non-chiral plasmonic nanoantennas. Nano. Lett. 14, 4426-4431 (2014)

28. Shaltout, A., Liu, J., Kildishev, A. \& Shalaev, V. Photonic spin Hall effect in gap-plasmon metasurfaces for on-chip chiroptical spectroscopy. Optica 2 860-863 (2015).

29. Pfeiffer, C. \& Grbic, A. Bianisotropic metasurfaces for optimal polarization control: Analysis and synthesis. Phys. Rev. Appl. 2, 044011 (2014).

30. Shaltout, A., Shalaev, V. \& Kildishev, A. Homogenization of bi-anisotropic metasurfaces. Opt. Express 21, 21941-21950 (2013).

31. Noginov, M. A. et al. Demonstration of a spaser-based nanolaser. Nature $\mathbf{4 6 0}$ 1110-1112 (2009)

32. Lu, Y. J. et al. Plasmonic nanolaser using epitaxially grown silver film. Science 337, 450-453 (2012).

33. Meng, X., Liu, J., Kildishev, A. V. \& Shalaev, V. M. Highly directional spaser array for the red wavelength region. Laser Photonics Rev. 8, 896-903 (2014).

34. Purcell, E., Torrey, H. \& Pound, R. Resonance absorption by nuclear magnetic moments in a solid. Phys. Rev. 69, 37-38 (1946).

35. Michler, P. et al. A quantum dot single-photon turnstile device. Science 290, 2282-2285 (2000)

36. Moreau, E. et al. Single-mode solid-state single photon source based on isolated quantum dots in pillar microcavities. Appl. Phys. Lett. 79, 2865-2867 (2001).

37. Khajavikhan, M. et al. Thresholdless nanoscale coaxial lasers. Nature $\mathbf{4 8 2}$ 204-207 (2012).

38. Baba, T., Fujita, M., Sakai, A., Kihara, M. \& Watanabe, R. Lasing characteristics of GaInAsP-InP strained quantum-well microdisk injection lasers with diameter of 2-10 $\mu \mathrm{m}$. IEEE Photonics Technol. Lett. 9, 878-880 (1997).

39. Baba, T. \& Sano, D. Low-threshold lasing and purcell effect in microdisk lasers at room temperature. IEEE J. Sel. Top. Quantum Electron. 9, 1340-1346 (2003).

40. Akahane, Y., Asano, T., Song, B. \& Noda, S. High- Q photonic nanocavity in a two-dimensional photonic crystal. Nature 425, 944-947 (2003).

41. Genevet, P. et al. Large enhancement of nonlinear optical phenomena by plasmonic nanocavity gratings. Nano. Lett. 10, 4880-4883 (2010).

42. Yoshle, T. et al. Vacuum Rabi splitting with a single quantum dot in a photonic crystal nanocavity. Nature 432, 200-203 (2004).

43. Engheta, N. An idea for thin subwavelength cavity resonators using metamaterials with negative permittivity and permeability. IEEE Antennas Wirel. Propag. Lett. 1, 10-13 (2002).

44. Caiazzo, M., Maci, S. \& Engheta, N. A metamaterial surface for compact cavity resonators. IEEE Antennas Wirel. Propag. Lett. 3, 261-264 (2004).

45. MacLeod, H. A. Thin-Film Optical Filters Ch. 8 (CRC Press, Florida, USA 2010).

46. Sun, S. et al. High-efficiency broadband anomalous reflection by gradient meta-surfaces. Nano. Lett. 12, 6223-6229 (2012). 
47. Pors, A., Nielsen, M. G., Eriksen, R. L. \& Bozhevolnyi, S. I. Broadband focusing flat mirrors based on plasmonic gradient metasurfaces. Nano. Lett 13, 829-834 (2013).

48. Pors, A., Albrektsen, O., Radko, I. P. \& Bozhevolnyi, S. I. Gap plasmonbased metasurfaces for total control of reflected light. Sci. Rep. 3, 2155 (2013).

49. Ameling, R., Dregely, D. \& Giessen, H. Strong coupling of localized and surface plasmons to microcavity modes. Opt. Lett. 36, 2218-2220 (2011).

50. Chen, W., Thoreson, M. D., Campus, M., Kildishev, A. V. \& Shalaev, V. M. Ultra-thin ultra-smooth and low-loss silver films on a germanium wetting layer on a germanium wetting layer. Opt. Express 18, 5124-5134 (2010).

51. Park, H. J., Xu, T., Lee, J. Y., Ledbetter, A. \& Guo, L. J. Photonic colour filters integrated with organic solar cells for energy harvesting. ACS Nano 5, 7055-7060 (2011).

52. D'Aguanno, G., Mattiucci, N., Alù, A. \& Bloemer, M. J. Quenched optical transmission in ultrathin subwavelength plasmonic gratings. Phys. Rev. B 83, 035426 (2011).

53. Zeng, B., Gao, Y. \& Bartoli, F. J. Ultrathin nanostructured metals for highly transmissive plasmonic subtractive colour filters. Sci. Rep. 3, 2840 (2013).

54. Hagag, M. A., Drachev, V., \& Kildishev, A. Characterizing NL response of metal-dielectric metasurfaces. In Proc. 2016 Conference on Lasers and ElectroOptics (IEEE, San Jose, CA, 2016).

\section{Acknowledgements}

A.M.S., J.K., A.B., and V.M.S. were supported by Air Force Office of Scientific Research (AFOSR) MURI grant FA9550-14-1-0389.A.V.K. would like to acknowledge support by DARPA/DSO Extreme Optics and Imaging (EXTREME) program, Award HR00111720032.

\section{Author contributions}

A.M.S. and J.K. developed the concept. A.M.S. designed and simulated the prototype structures. J.K. carried out the multi-layer fabrication of the devices. J.K and A.M.S. performed the experiments. A.V.K. guided the design of optical cavities with embedded metasurfaces. A.V.K., A.B., and V.M.S. oversaw the project and provided the facilities. All the authors contributed and approved the manuscript.

\section{Additional information}

Supplementary Information accompanies this paper at https://doi.org/10.1038/s41467018-05034-6.

Competing interests: The authors declare no competing interests.

Reprints and permission information is available online at http://npg.nature.com/ reprintsandpermissions/

Publisher's note: Springer Nature remains neutral with regard to jurisdictional claims in published maps and institutional affiliations.

\begin{abstract}
(c) (i)
Open Access This article is licensed under a Creative Commons Attribution 4.0 International License, which permits use, sharing, adaptation, distribution and reproduction in any medium or format, as long as you give appropriate credit to the original author(s) and the source, provide a link to the Creative Commons license, and indicate if changes were made. The images or other third party material in this article are included in the article's Creative Commons license, unless indicated otherwise in a credit line to the material. If material is not included in the article's Creative Commons license and your intended use is not permitted by statutory regulation or exceeds the permitted use, you will need to obtain permission directly from the copyright holder. To view a copy of this license, visit http://creativecommons.org/ licenses/by/4.0/.
\end{abstract}

(C) The Author(s) 2018 